\title{
Value of window technique in diagnosis of the ground glass opacities in patients with non-small cell pulmonary cancer
}

\author{
GANG YAO \\ Department of Radiology, Zaozhuang Municipal Hospital, Zaozhuang, Shandong 277100, P.R. China
}

Received April 22, 2016; Accepted September 12, 2016

DOI: $10.3892 / \mathrm{ol} .2016 .5133$

\begin{abstract}
The aim of the present study was to examine the value of window technique in qualitative diagnosis of the ground glass opacities (GGO) in patients with non-small cell pulmonary cancer. A total of 124 clinically suspected pulmonary cancer patients were analyzed retrospectively. The lesions were affirmed by puncture biopsy, and were GGO on pulmonary window while were invisible on mediastinal window. Sixty-four multi-detector spiral computed tomography with the window width and window level of 1,500 Hounsfield units (HU) and $-450 \mathrm{HU}$ on pulmonary window, while the window width and window level of 400 and $40 \mathrm{HU}$ on mediastinal window, was used in the study. The window adjustment technique was used to analyze the window width and window level of lesion on pulmonary window and mediastinal window, for searching invisible threshold on 3-megapixel medical displays. The diagnostic accuracy and the cut-off value were compared on receiver operating characteristic (ROC) curve. The results showed that the window width and window level on pulmonary window and mediastinal window of malignant lesions were significantly less than those of benign ones $(\mathrm{P}<0.05)$. The cut-off value on pulmonary window was the window width and window level of 1,300 and $-220 \mathrm{HU}$, the area under the ROC was 0.830 [sensitivity was $72.5 \%$, specificity was $84.3 \%$; $95 \%$ confidence interval (CI), 0.712-0.945]. The cut-off value on mediastinal window was the window width and window level of 360 and $30 \mathrm{HU}$, and the area under the ROC was 0.623 (was $62.0 \%$, specificity was $55.7 \%$; 95\% CI, 0.541-0.745). In conclusion, the window technique has high sensitivity and accuracy in qualitative diagnosis of the GGO.
\end{abstract}

\section{Introduction}

Ground glass opacities (GGO) refers to the lesions manifested as blur and high-density image on pulmonary window, while

Correspondence to: Dr Gang Yao, Department of Radiology, Zaozhuang Municipal Hospital, 41 Longtou Road, Zaozhuang, Shandong 277100, P.R. China

E-mail: yao_gang1212@163.com

Key words: window technology, window width, window level, non-small cell pulmonary cancer, ground glass opacities, receiver operating characteristic, cut-off value these lesions were either invisible or only the solid portion was visible on mediastinal window. The texture of vessel and bronchus of lesion were clear. GGO were closely associated with pulmonary cancer, and were regarded as precancerous lesions (1). However, the lesions were also found in inflammation, infection and tuberculosis (2). Thus, early qualitative diagnosis of the GGO is very important for the treatment of diseases.

The use of pre-set parameter to obtain GGO images has some limitations with regard to qualitative diagnosis (3). The operational level of technical personnel, scanning parameter setting, the accuracy of computed tomography (CT) values, and the quality of the system were considered to be important factors influencing qualitative diagnosis (4). The window technique is a type of amplification technology that takes advantage of the gray-scale function in computer software to enlarge the visual gray-scale identification range (5). It can adjust the window width and window level of lesion on pulmonary window and mediastinal window continuously and dynamically, to obtain the best range of gray-scale, and compare the window characteristics of different GGO to obtain more sensitive and accurate quantitative data.

The aim of the present study was to examine the value of window technique in qualitative diagnosis of the GGO in patients with non-small cell pulmonary cancer. The results showed that, the window width and window level on pulmonary window and mediastinal window of malignant lesions were significantly less than those of benign ones.

\section{Patients and methods}

Objects. In total, 124 clinically suspected pulmonary cancer patients were retrospectively analyzed between January, 2012 and January, 2016. The lesions were affirmed by puncture biopsy, and were GGO on pulmonary window but invisible on mediastinal window. The following conditions were excluded: a clear diagnosis of tumor, recent history of pneumonia, pulmonary tuberculosis, thoracic injury, surgery and history of radiation exposure, poor quality image. The Zaozhuang Municipal Hospital (Shandong, China) Ethics Committee approved the study. Informed consent was obtained from the patients or their family members.

Of the 124 cases, 93 patients had tumor, and 31 had benign lesions $(25.0 \%)$. Of the 93 tumor lesions, 40 were squamous carcinoma, 49 were adenocarcinoma, and 4 were others. There 
were 37 atypical hyperplasia, 35 were in situ, and 21 were invasive lesions. There were 52 lesions with round or ovoid shape, and 41 were irregular in shape. The average diameter of the lesions was $0.6 \pm 0.3 \mathrm{~cm}$ (range, $0.3-1.0 \mathrm{~cm}$ ), with 46 cases having left pulmonary and 47 cases right pulmonary lesions. The patients with tumors included 52 males and 41 females, and the average age was $56.9 \pm 14.7$ years $(6-48$ years). If the 31 benign lesions, 23 cases were pneumonia, and 8 cases were tuberculosis, while of the 31 cases 20 were round or ovoid shaped, and 11 irregular in shape. The average diameter of the lesions was $0.5 \pm 0.4 \mathrm{~cm}$ (range, $0.2-1.3 \mathrm{~cm}$ ), 13 lesions were in the left lung, and 18 were in the right lung. The patients with benign lesions included 17 males and 14 females, and the average age was $54.6 \pm 15.2$ years ( $37-73$ years). The difference of gender, age, pathological changes, morphology and diameter of comparison between the two groups was not significantly different.

CT scanning methods. Siemens Sensation 64 multi-detector spiral CT (Siemens AG, Munich, Germany) was used, with a parameter of pitch 1.2 , collimation width $128 \times 0.6 \mathrm{~mm}$, scanning thickness $5 \mathrm{~mm}$, tube voltage $120 \mathrm{kV}$, automatic tube current, reconstruction thickness $1 \mathrm{~mm}$, window width 1,500 Hounsfield units (HU) and window level $-450 \mathrm{HU}$ on pulmonary window, window width $400 \mathrm{HU}$ and window level $40 \mathrm{HU}$ on mediastinal window. A plain scan was used without oral contrast material.

For window technique, two radiology doctors analyzed the images in a blinded manner. The window width was adjusted constantly until the lesions were invisible with the fixed pulmonary window level $(-450 \mathrm{HU})$ on 3-megapixel medical displays, and then the pulmonary window width was fixed $(150 \mathrm{HU})$, and the window level was adjusted constantly until the lesions were invisible. The window width was adjusted constantly until the lesions were invisible with the fixed mediastinal window level $(40 \mathrm{HU})$, and then the mediastinal window width $(400 \mathrm{HU})$ was fixed, and the window level adjusted constantly until the lesions were invisible. Multi-plane reorganization observation was used to obtain the average. Those above the upper limitation gray-scale range were enhanced to white, and those below the upper limitation were compressed to black. $M-(W / 2)-M+(W / 2)$ was used to represent (M, window level; $\mathrm{W}$, window width). $\mathrm{M}=\left(\mathrm{CT}_{\max }-\mathrm{CT}_{\min }\right) / 2, \mathrm{~W}=\mathrm{CT}_{\max }-\mathrm{CT}_{\min }$.

Statistical analysis. Data were analyzed using SPSS 19.0 statistical software (Chicago, IL, USA). The quantitative data were expressed as means \pm standard deviation, and the difference of groups was compared by t-test. Qualitative data were expressed as the number of cases or percentage (\%), and the difference of groups was compared using the $\chi^{2}$ test. The area under the receiver operating characteristic (ROC) curve represented the diagnostic accuracy, and difference with $\mathrm{P}<0.05$ was considered to indicate a statistically significant difference.

\section{Results}

Comparison of window width and window level. The window width and window level on the pulmonary and mediastinal windows of malignant lesions was significantly less than those of benign ones $(\mathrm{P}<0.05)$, as shown in Table I.
Table I. Comparison of window width and window level (HU).

\begin{tabular}{lcrrrr}
\hline \multirow{2}{*}{ Groups } & \multicolumn{2}{c}{ Pulmonary window } & & \multicolumn{2}{c}{ Mediastinal window } \\
\cline { 2 - 3 } & Width & Level & & Width & Level \\
\hline Malignant & $1,356 \pm 53$ & $-210 \pm 35$ & & $353 \pm 45$ & $26 \pm 10$ \\
Benign & $2,124 \pm 67$ & $-546 \pm 43$ & & $652 \pm 52$ & $63 \pm 20$ \\
t-test & 7.632 & 6.549 & & 7.541 & 7.835 \\
P-value & $<0.001$ & $<0.001$ & & $<0.001$ & $<0.001$ \\
\hline
\end{tabular}

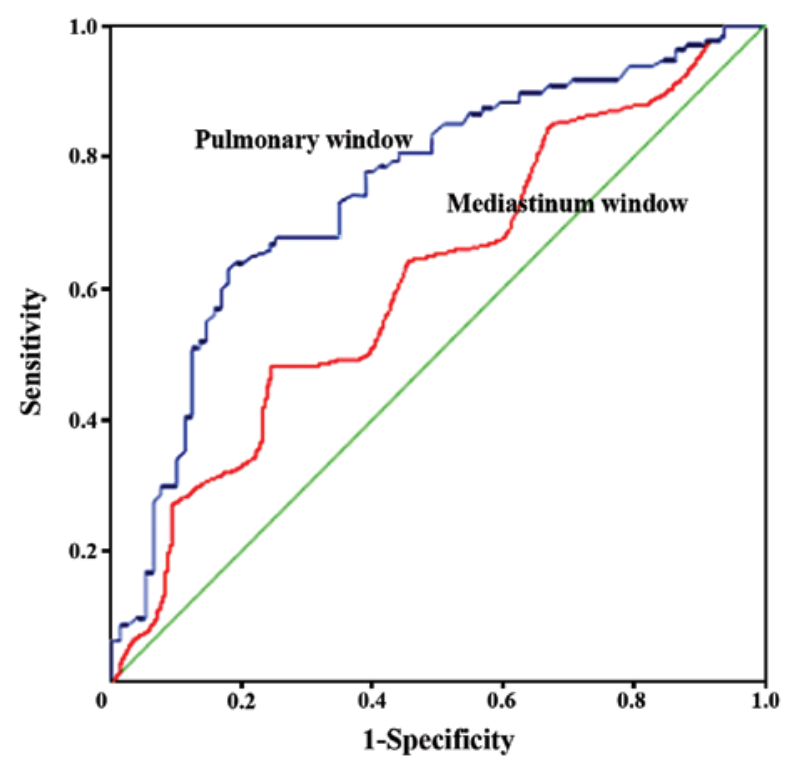

Figure 1. ROC analysis of window technology in the diagnosis of malignant lesions. ROC, receiver operating characteristic.

ROC analysis. ROC was applied to analyze the usage of the pulmonary and mediastinum window technique in the diagnosis of malignant lesions. The cut-off value on pulmonary window was the window width and window level of 1,300 and -220 HU, the area under the ROC was 0.830 [sensitivity was $72.5 \%$, specificity was $84.3 \%$; $95 \%$ confidence interval (CI), 0.712$0.945]$. The cut-off value on the mediastinal window was the window width and window level of 360 and $30 \mathrm{HU}$, and the area under the ROC was 0.623 (sensitivity was $62.0 \%$, specificity was 55.7\%; 95\% CI, 0.541-0.745), as shown in Fig. 1.

\section{Discussion}

The window width mainly affected the contrast of the image. The large window width multiplies the levels of the image, reduces the contrast and deteriorates the detail display, which is suitable for distinguishing the structure with large density difference (6). The small window width decreased the levels of the image, enhanced the contrast, and optimized the detail display, which was suitable for distinguishing the structure with small density difference. The window level mainly affected the image contrast. The high window darkened the image, and the window was equivalent to the center of the gray-scale (7). The best window width and window level is necessary for setting the standard window, and was manifested 
as 35 picture/sheet at the center of the image. Subsequently, the window width and window level was adjusted slightly and each part of 4-6 images was captured (8). The narrow window technique was suitable for lesions with small density difference and unclear boundary (9). Thus, lesions with slight density differences or a large range and rich layers need to use the wide window technique (10).

The development of vessels and bronchi pulmonary window have a greater interference to the qualitative diagnosis of GGO. Early pulmonary cancer mainly originates in bronchial epithelium, with the involvement of centrilobular bronchi or centrilobular arterial nodules, and located around the blood vessels, around or through the minimum pulmonary arteries that CT show. The adjustment of the pulmonary window width and window level can distinguish the imaging range of GGO from blood vessels and bronchi to improve the accuracy of clinical diagnosis $(11,12)$. The common parameter is window width of 800-1,000 HU, window level of $-700 \mathrm{HU}$, and upper bound of -200 HU. Fat, fluid, soft tissue and bone were located out of the window by analyzing the density, and in sharp contrast with the pulmonary tissue, which was not suitable to display the characteristics of lesions and the inflammation. Additionally, it was difficult to distinguish the lesions in the center with vascular section. The window width and window level on pulmonary window and mediastinal window of malignant lesions was significantly less than those of benign ones. In the malignant group, the average window width and window level was of 1,356 and $-210 \mathrm{HU}$ on pulmonary window. The diameter of the GGO, the size of solid or pathological invasive component, and the composition of ground glass proportion have great limitations to qualitative diagnosis (13). The tumors can be divided into gas type and solid type according to mediastinal window width and window level technology. The gas type has good prognosis and applicable value $(14,15)$. The mediastinal window cannot display GGO lesions well, so the mediastinal window technique remains in limited use.

The adjustment of the pulmonary window width and window level can highlight the boundaries of the tissues and the appearing and disappearing range of $\mathrm{CT}$, to improve the accuracy of clinical diagnosis. The prognosis was assessed based on the size of tumor alone, but disagreement also exist $(16,17)$.

In conclusion, the cut-off value on pulmonary window was the window width and window level of 1,300 and $-220 \mathrm{HU}$, the area under the ROC was 0.830 (sensitivity was $72.5 \%$, specificity was $84.3 \%$; $95 \%$ CI, 0.712-0.945). The cut-off value on the mediastinal window was the window width and window level of 360 and $30 \mathrm{HU}$, and the area under the ROC was 0.623 (sensitivity was $62.0 \%$, specificity was $55.7 \%$; $95 \%$ CI, 0.541 0.745). The diagnosis sensitivity, specificity and accuracy of pulmonary window were better than those of mediastinal window. However, the frequent adjustment of window technology will lead to visual fatigue, and increase workload. Further exploration of a more simple and higher quantitative inspection method is required.

\section{References}

1. Min JH, Lee HY, Lee KS, Han J, Park K, Ahn MJ and Lee SJ: Stepwise evolution from a focal pure pulmonary ground-glass opacity nodule into an invasive lung adenocarcinoma: an observation for more than 10 years. Lung Cancer 69: 123-126, 2010.

2. Pedersen JH, Saghir Z, Wille MM, Thomsen LH, Skov BG and Ashraf $\mathrm{H}$ : Ground-glass opacity lung nodules in the era of lung cancer ct screening: radiology, pathology, and clinical management. Oncology (Williston Park) 30: 266-274, 2016.

3. Saji H, Matsubayashi J, Akata S, Shimada Y, Kato Y, Kudo Y, Nagao T, Park J, Kakihana M, Kajiwara N, et al: Correlation between whole tumor size and solid component size on high-resolution computed tomography in the prediction of the degree of pathologic malignancy and the prognostic outcome in primary lung adenocarcinoma. Acta Radiol 56: 1187-1195, 2015.

4. Lee HY and Lee KS: Ground-glass opacity nodules: histopathology, imaging evaluation, and clinical implications. J Thorac Imaging 26: 106-118, 2011.

5. Murakawa T, Konoeda C, Ito T, Inoue Y, Sano A, Nagayama K and Nakajima J: The ground glass opacity component can be eliminated from the T-factor assessment of lung adenocarcinoma. Eur J Cardiothorac Surg 43: 925-932, 2013.

6. Liu B, Mi RH, Wei XD, Chen L, Ai H, Yuan FF, Yin QS and Song YP: Clinical analysis of grand glass opacity on CT as the first change of lung infection accompanied by hematologic malignancies. Zhonghua Yi Xue Za Zhi 96: 163-166, 2016 (In Chinese).

7. Nam KB, Kim TJ, Park JS, Chung MJ and Lee KW: Long-term follow-up results from PET/CT surveillance after surgical resection of lung adenocarcinoma manifesting as ground-glass opacity. Medicine (Baltimore) 95: e2634, 2016.

8. Lee HN, Kim MY, Koo HJ, Kim SS, Yoon DH, Lee JC and Song JW: and Thin-Section CT: Thin-section ct characteristics and longitudinal CT follow-up of chemotherapy induced interstitial pneumonitis: a retrospective cohort study. Medicine (Baltimore) 95: e2460, 2016.

9. Cha MJ, Lee KS, Kim HS, Lee SW, Jeong CJ, Kim EY and Lee HY: Improvement in imaging diagnosis technique and modalities for solitary pulmonary nodules: from ground-glass opacity nodules to part-solid and solid nodules. Expert Rev Respir Med 10: 261-278, 2016.

10. Chen CY, Chen CH, Shen TC, Cheng WC, Hsu CN, Liao CH, Chen CY, Hsia TC, Liao WC, Tu CY, et al: Lung cancer screening with low-dose computed tomography: experiences from a tertiary hospital in Taiwan. J Formos Med Assoc 115: 163-170, 2016.

11. Jiang G and Xie D: Early-stage lung cancer manifested as ground-glass opacity. Zhonghua Wai Ke Za Zhi 53: 790-793, 2015 (In Chinese).

12. Bak SH, Lee HY, Kim JH, Um SW, Kwon OJ, Han J, Kim HK, Kim J and Lee KS: Quantitative CT scanning analysis of pure ground-glass opacity nodules predicts further CT scanning change. Chest 149: 180-191, 2016.

13. Ichikawa K, Kobayashi T, Sagawa M, Katagiri A, Uno Y, Nishioka R and Matsuyama J: A phantom study investigating the relationship between ground-glass opacity visibility and physical detectability index in low-dose chest computed tomography. J Appl Clin Med Phys 16: 5001, 2015.

14. Ikehara M, Saito H, Yamada K, Oshita F, Noda K, Nakayama H, Masui K, Kameda Y, Komase Y and Miyazawa T: Prognosis of small adenocarcinoma of the lung based on thin-section computed tomography and pathological preparations. J Comput Assist Tomogr 32: 426-431, 2008.

15. Honda T, Kondo T, Murakami S, Saito H, Oshita F, Ito H, Tsuboi M, Nakayama H, Yokose T, Kameda Y, et al: Radiographic and pathological analysis of small lung adenocarcinoma using the new IASLC classification. Clin Radiol 68: e21-e26, 2013.

16. Yang F, Chen H, Xiang J, Zhang Y, Zhou J, Hu H, Zhang J and Luo X: Relationship between tumor size and disease stage in non-small cell lung cancer. BMC Cancer 10: 474, 2010.

17. Patz EF Jr, Rossi S, Harpole DH Jr, Herndon JE and Goodman PC: Correlation of tumor size and survival in patients with stage IA non-small cell lung cancer. Chest 117: 1568-1571, 2000. 